\title{
Hyperbaric oxygen treatment increases intestinal stem cell proliferation through mTORc1 signaling in Mus musculus
}

\section{ignacio casanova}

Universidad de Chile https://orcid.org/0000-0001-9502-570X

\section{Isaac Peña-Villalobos}

University of Chile: Universidad de Chile

\section{David Arancibia-Altamirano}

University of Chile: Universidad de Chile

\section{Pablo Lois}

Mayor University: Universidad Mayor

Verónica Palma ( $\nabla$ vpalma@uchile.cl) University of Chile: Universidad de Chile

\section{Research}

Keywords: Intestinal stem cells, Hyperbaric oxygen treatment, mTORc1, proliferation, rapamycin

Posted Date: November 20th, 2020

DOl: https://doi.org/10.21203/rs.3.rs-108513/v1

License: (c) (i) This work is licensed under a Creative Commons Attribution 4.0 International License. Read Full License 
1 Hyperbaric oxygen treatment increases intestinal stem cell proliferation through mTORc1 signaling

2 in Mus musculus

3

4

5 Ignacio Casanova-Maldonado ${ }^{1}$, Isaac Peña-Villalobos ${ }^{1}$, David Arancibia ${ }^{1}$, Pablo Lois ${ }^{1,2}$ and

6 Verónica Palma ${ }^{1 *}$

7

8

1. Laboratory of Stem Cells and Developmental Biology, Faculty of Sciences. Universidad de Chile. Santiago, Chile.

10

11

12

13

14

15

16

17

18

19

20

21

22

23

24

25

26

27

28

29

30

8

* Corresponding Author: Verónica Palma (vpalma@uchile.cl), Phone number (56-2) 2978 7221 . Las

Encinas 3370. Milenio Building Floor 3, Ñuñoa, Santiago, Chile. 7800024

2. Present address: Postgraduate in Education Department; Faculty of Humanities, Universidad Mayor

2

3

4

Encinas 3370. Milenio Building Floor 3, Nuño, Santiago, Chile. 7800024

(8)

8

9

0

1

2

3

4

5

6

7

8

9


33 Abbreviations: Hyperbaric oxygen treatment (HBOT), mTOR complex 1 (mTORc1), intestinal 34 stem cells (ISCs), caloric restriction (CR), columnar base cells (CBC), Bromodeoxyuridine (BrdU), 35 phospho-Histone H3 (PHH3), cytochrome c oxidase (COX), citrate synthase (CS), 36 immunofluorescence (IF), immunohistochemistry (IHC)

37 
41 Background: Hyperbaric oxygen treatment (HBOT) has been used for more than a decade to treat

42 diverse diseases like diabetic foot ulcers and ischemic injuries. More recently, HBOT has been

43 reported to modulate proliferation of neural and intestinal stem cell populations, but the molecular

44 mechanisms underlying these effects are not completely understood.

45 Objective: In this study we aimed to determine HBOT stem cell modulation by evaluating in 46 particular the role of the mTOR complex 1 (mTORc1), a key regulator of cell metabolism that 47 modifies its activity depending on oxygen levels, as a potential mediator of HBOT in murine intestinal 48 stem cells (ISCs).

49 Methods: Mouse were exposed to 10 or 20 HBOT sessions and the proliferation of the ISCs were 50 analyzed by immunofluorescence or immunohistochemistry using the specific ISCs marker, Olfm4. 51 The regulation of HBOT and mTORc1 pathway was analyzed through S6K1 phosphorylation by 52 western-blot and through the inhibition by rapamycin.

53 Results: We discovered that acute HBOT can increase proliferation of ISCs in a synchronous fashion 54 without affecting the animal's oxidative metabolism. Noteworthy, the mTORc1 inhibitor rapamycin 55 also increases the proliferation of ISCs. This effect has been attributed to its capacity to mimic a 56 caloric restriction (CR). Interestingly, the combination of HBOT and rapamycin does not have a 57 synergic effect. Nevertheless, HBOT can recover rapamycin induced mTORc1 inhibition, possibly 58 acting through a competitive modulation on mTORC1.

59 Conclusions: Collectively, our results suggest that HBOT proliferative effect on ISCs is modulated 60 by mTORc1 signaling representing a promising new approach to treat intestinal conditions. 
64 Hyperbaric oxygen treatment (HBOT) consists in administration of oxygen at high pressures. HBOT

65 works under Henry's law that states that the amount of gas dissolved in a liquid is proportional to the

66 partial pressure that the gas applies over such liquid. As a result, the amount of oxygen dissolved in

67 blood increases, which causes a systemic effect by enriching tissues in oxygen through the bloodstream.

HBOT is widely used as an adjuvant treatment to treat diabetic foot ulcers (Kalani et al; 2002), brain injuries (Zhang et al; 2010 and Bennett et al; 2012) and to promote wound healing (Eskes et al; 2011

71 and Kranke et al; 2015 and Peña-Villalobos et al; 2018). Recent studies have proposed that its effects 72 on wound healing occurs due modulation of HIF-1 $\alpha$ (Sunkari et al; 2015) and Vascular Endothelial 73 Growth Factor (VEGF) (Lee et al; 2006) signaling pathways. Nonetheless, the mechanisms are under 74 continuous debate and still far from being fully understood.

75 In the last decade, studies have focused on stem cells as cellular targets of HBOT, revealing positive effects on proliferation and regeneration on both neural (Zhang et al; 2011) and mesenchymal stem

77 cell populations (Dhar et al; 2012). An interesting niche for studying the effects of HBOT on stem 78 cell proliferation is the intestinal epithelium, the most vigorously self-renewing

79 tissue of adult mammals (Snippert et al; 2010). Within this epithelium, intestinal stem cells (ISCs) 80 reside at the base of the crypts of Lieberkühn, allowing the generation of absorptive or secretory 81 progenitors who migrate toward the tip of the villus (Barker et al; 2014), regenerating the small 82 intestine every 3 days in mice (Totafurno et al; 1987 and Clevers; 2013). The crypts of Lieberkühn 83 have been well studied and characterized in terms of their cellular composition. ISCs are also named 84 columnar base cells (CBC) for their position in the base of the crypts and can be identified by the 85 expression of specific markers like Lgr5 (a membrane protein) or Olfm4 (a cytoplasmatic protein). 86 CBCs give rise to another functionally distinct population of stem cells, called +4 (due to their 87 position in the crypt) or emergency stem cells. The complex nature of ISCs is becoming clear in recent 
years, revealing a hierarchy between Lgr5+ and +4 under homeostasis and stress responses (De Mey \& Freund; 2013 and Basak et al; 2017).

The homeostatic renewal in the intestinal epithelium is commanded by oxidative metabolism and guided by the mTOR complex 1 (mTORc1), a macromolecular nutrient- and growth factor-responsive kinase (Yilmaz et al; 2012 and Sampson et al; 2015). The kinase1 is also able to integrate a response to transcription factors (Igarashi et al; 2016) and oxygen (Schieke et al; 2006 and Saxton \& Sabatini; 2017). mTORc1 signaling involves the activation of many proteins like 4E-BP1 and S6K1 through phosphorylation, regulating cellular anabolic growth and proliferation (Fingar et al; 2002). A plethora of studies have shown that this complex can be inhibited by the administration of rapamycin, an allosteric inhibitor (Benjamin et al; 2011), preventing the formation of the complex and therefore downregulating the signaling pathway (Fingar et al; 2004). Furthermore, rapamycin has been used to mimic the effects of a caloric restriction (CR), resulting in an increase of lifespan in different species like yeast, mice and humans (Cox et al; 2009, Dai et al; 2014 and Wang et al; 2017).

The regulatory mechanisms that control ISCs in response to HBOT are just beginning to be explored. Here we analyzed the effects of acute HBOT on the ISCs proliferation rates of Mus musculus small intestine and its modulation by mTORc1. First, we established the number of HBOT sessions required to promote a positive effect on ISCs proliferation. Secondly, we sought to understand the complex relation between oxygen tension and proliferation, mediated by the mTOR complex. Our results show that animals treated for 10 days with HBOT present a trend to increase ISCs proliferation. However, animals treated with a daily HBOT session for 20 consecutive days show an increase in proliferation in a synchronous fashion among crypts. Noteworthy, although animals treated with rapamycin also increase the number of ISC proliferating by crypt, mimicking the effects of a CR (Cox et al; 2009 and Dai et al; 2014), a combined treatment with HBOT and rapamycin did not increase ISC proliferation. We speculate that the latter is probably due a competition for mTORc1. Strikingly, we found that HBOT can recover the inhibition of rapamycin over mTORC1. Overall, our findings suggest that 
113 HBOT can replicate the effects of a CR, stimulating proliferation of ISCs. As such, we propose that 114 HBOT could be a potential adjuvant treatment for intestinal injuries and pathologies.

115 
117 Animals

118 Forty-six adult (3 months old) males of the BALB/c strain of Mus musculus were obtained from the 119 central animal housing facilities at the Faculty of Sciences. All animal procedures were in accordance 120 with the Chilean legislation and were approved by Institutional Animal Care and Use Committee 121 (CICUA) at the Universidad de Chile.

122 To analyze the effects of HBOT on ISCs proliferation we generated four groups: Control group for 123 ten days (C10D), HBOT group for ten days (H10D), Control group for twenty days (C20D) and 124 HBOT for twenty days (H20D) (Table I). Each group was formed by five animals, considering no 125 siblings in the same group. They were housed with their siblings but properly identified and kept in 126 a temperature-controlled room, maintained at $25^{\circ} \pm 1{ }^{\circ} \mathrm{C}$ in a $\mathrm{LD}=12: 12$ cycle, with food and water 127 ad libitum.

128 In order to explore the relationship of HBOT with mTORC1 signaling we generated another set of 4 129 experimental groups: 2 Groups without HBOT sessions but injected with rapamycin (Rapa Ctrl) or 130 with DMSO (vehicle of rapamycin) (DMSO Ctrl), and 2 groups treated with HBOT injected with 131 rapamycin (RAPA HBOT) or with DMSO (DMSO HBOT) (Table II). All groups were treated for 20 132 consecutive days. Each group included five animals, maintained in the same conditions as described 133 above.

134 Table I: Animal groups generated for analysis of HBOT impact on ISCs proliferation.

\begin{tabular}{|c|c|c|}
\hline \multicolumn{3}{|c|}{ ISCs proliferation } \\
\hline & 10 Days & 20 Days \\
\hline Control & C10D & C20D \\
\hline HBOT & H10D & H20D \\
\hline
\end{tabular}


137 pathway and ISCs proliferation.

138

139

140

141

142

143

144

145

146

147

148

149

150

151

152

153

154

155

156

\begin{tabular}{|c|c|c|}
\hline \multicolumn{3}{|c|}{ mTOR and ISCs } \\
\hline & Control & HBOT \\
\hline & DMSO & DMSO \\
DMSO & Ctrl & HBOT \\
\hline rapamycin & Rapa Ctrl & Rapa HBOT \\
\hline
\end{tabular}

\section{HBOT sessions}

HBOT was performed in a $19.56 \mathrm{~L}$ experimental chamber at $25^{\circ} \mathrm{C}$ (Osorio Hermanos \& Cia. Ltd., Quillota, Chile). Animals were placed in individual cages, then the remaining air inside the chamber was replaced with $100 \% \mathrm{O}_{2}$ while the pressure was increased for 15 minutes until 2.0 ATA (absolute atmospheres) were reached. The latter conditions were maintained for $1.0 \mathrm{~h}$ followed by decompression from 2.0 ATA to atmospheric pressure gradually for other 15 min (figure 1A). Therefore, each session took 1.5 h (Dave et al; 2003 and Peña-Villalobos et al; 2018).

\section{Rapamycin administration}

Rapamycin (Calbiochem, 553210) was dissolved in DMSO (5 mg/125 $\mu \mathrm{l})$ and diluted in PBS 1X 1:100 v/v. Every other day rapamycin was injected intraperitoneally $(2 \mathrm{mg} / \mathrm{kg})$, always at the same hour and before starting the HBOT session.

\section{Intestinal tissue dissection}

After the last HBOT session animals were injected intraperitoneally with $800 \mu \mathrm{l}$ of $20 \mathrm{mg} / \mathrm{ml} \mathrm{BrdU}$ (Sigma-Aldrich). One hour later the animals were sacrificed by cervical dislocation. Immediately the entire digestive tract was dissected on a cooled surface and the intestine contents were gently removed mechanically. Next, the first third of the small intestine (duodenum) was cut longitudinally and used for histological analysis. This segment was fixed in $4 \%$ PFA (Sigma-Aldrich) for $2 \mathrm{~h}$ at $4{ }^{\circ} \mathrm{C}$ followed by dehydration overnight (O.N) in 30\% sucrose (Merck) solution. Tissues were embedded in OCT 
157 (Tissue-Tek), placed in a disposable vinyl specimen mold (Tissue-Tek Cryomold) and stored at $-80^{\circ} \mathrm{C}$ 158 until further use.

\section{Immunostaining}

160 To realize immunofluorescence (IF) and immunohistochemistry (IHC) we followed the exact same 161 protocol of Peña-Villalobos et al. (2018). Briefly, slides of either 14 and $7 \mu \mathrm{m}$ thickness were prepared 162 with the epitope-unmasking protocol and incubated overnight at $4^{\circ} \mathrm{C}$ with $1: 100$ monoclonal mouse 163 Anti-Bromodeoxyuridine Clone Bu20a (BrdU, Dako, M0744) primary antibody or 1:400 monoclonal 164 rabbit Anti-Olfm4 (Cell Signaling, 39141). For IF, we used 1:500 goat anti mouse Alexa 555 (Life 165 technology, A21424) as a secondary antibody to BrdU and 1:500 goat anti rabbit Alexa 488 (Life 166 technology, A11034) as a secondary to Olfm4. DAPI (Invitrogen) was used to label nuclei. For IHC, 167 we used hematoxylin/eosin (H\&E) and DAB staining as described in Peña-Villalobos et al. (2018). 168 To analyze the IHC, an optical microscope (Olympus BX51) at 40X and 100X equipped with a digital 169 camera (Moticam 2500) was used whereas for IF a confocal microscope (Zeiss 710) was used. Z170 stacks and image analysis was made by ImageJ and FIJI programs. Figures were created by 171 Biorender.com.

Enzyme Assays

173 The duodenum was homogenized in 10 volumes of phosphate buffer $0.1 \mathrm{M}$ with EDTA $0,002 \mathrm{M}(\mathrm{pH}$ 174 7.3) with an Ultra Turrax homogenizer $(20,000 \mathrm{rpm})$ on ice. Samples were sonicated for 45 seconds 175 on ice using an Ultrasonic Processor VCX 130. Cellular debris was removed by centrifugation for 15 $176 \min$ at $12,000 \mathrm{G}$ at $4^{\circ} \mathrm{C}$. Then the supernatant was isolated and used for determination of protein 177 concentration by Bradford method.

178 Citrate Synthase and Cytochrome C Oxidase activity were determined spectrophotometrically 179 according to Peña-Villalobos et al. 2018. 
182 Homogenized duodenums, stored at $-80^{\circ} \mathrm{C}$ with proteases and phosphatases inhibitors, were left on

183 ice for $1 \mathrm{~h} .50 \mu \mathrm{g}$ of the sample was loaded on acrylamide gel posterior to Lowry quantification (550 $184 \mathrm{~nm}$; Tecan M200) and then transferred to a nitrocellulose film. The film then was treated with BSA $1855 \%$ in TBS-T 0,1\% for an hour, washed in PBS and incubated for $12 \mathrm{~h}$ with anti-phospho p70 S6K1 186 (Thr389) (1:1000, Cell signaling) or anti-p70 S6K1 (1:1000, Cell signaling) and anti- $\beta$ actine (1: 187 5000, Cell Signaling). For protein detection we used Rabbit anti-mouse HRP (1:5000; Cell signaling) 188 189 190 191 to identify p70 S6K1 and p70 S6K1 phosphorylated and Mouse anti- $\beta$ actine (1:5000, Cell Signaling) with luminol. X-ray films were obtained after $1 \mathrm{~h}$ exposition in dark room for S6K1 and S6K1-P, while $\beta$ actine detection last 10 seconds of exposition.

\section{Statistical analysis}

The number of positive labeled cells per villus of each crypt were scored by two independent counters. To determinate if HBOT has effects on the proliferation dynamics of ISCs we counted the number of BrdU+ cells in 36 randomly chosen crypts from the first third of the intestine of each individual ( $\mathrm{n}=3$ per treatment). For IF, ISCs positive cells were considered only if they expressed the specific marker Olfm4 (Olfm4+ cells). Permutation test was performed in R software, using a permutation test script of 1000 permutation for each analysis. Data from mTOR inhibition by rapamycin and western blot were also analyzed by permutation test. To analyze the distribution of the data we used a Kolmogorov-Smirnov test. As for the first experiment (HBOT for either 10 or 20 days) we considered a Bonferroni correction, having an $\alpha=0.0125$. 
204 Stem cells in vivo reside in a dynamic and specialized microenvironment, the so- called niche. The

205

206

207

208

209

210

211

212

213

214

215

216

217 by calculating the stabilization coefficient (figure 1K) for each group. H20D was the only group that

218 shows clear differences. To evaluate if this synchronization in proliferation was generated by a 219 stem cell niche is influenced by a variety of factors including physical and metabolic parameters. Oxygen tension is known to be an important cellular input modulating stem cell self-renewal and differentiation potential. Thus, many stem cells, including ISCs, respond actively to changes in oxygen tension.

ISCs are located at the bottom of the intestinal crypt and can be identified as Olfm4+ cells, revealing a co-distribution with the BrdU label (figure $1 \mathrm{~B}-\mathrm{E}$ ). To determine the proliferation effects of HBOT on ISCs, we evaluated the number of BrdU+ cells per crypt in all experimental groups. Only small intestines from mice treated with HBOT for 20 days (H20D) show an increment in the number of BrdU+ cells (figure $1 \mathrm{~F}-\mathrm{J}$ ). Interestingly, another cell population, located at near position to the intestinal crypts, also exhibited BrdU staining (figure 1E). Hence, we cannot discard that HBOT has effects on another cell population, besides the Olfm4+ CBC pool.

HBOT increased proliferation of cells within the crypts in a synchronous way. This was determined possible arrest in the cell cycle due to treatment, we analyzed the ratio between BrdU and PHH3 positive cells in intestinal samples from C20D group or H20D group (data not shown). We did not find differences in these ratios. Hence, we conclude that HBOT does not generate this synchronization of proliferation due to a cell cycle arrest in ISCs.

\section{HBOT has an accumulative effect in time}

The frequency distribution from groups treated with H10D was different from its control C10D despite not showing differences in the numbers of BrdU+ cells per crypts. When we analyzed the 
226 frequency distribution for each group (figure 2), we found that only control groups showed no 227 differences $(\mathrm{p}=0.796)$. Interestingly, the frequency distribution from H10D was different, both from 228 the control groups and $\mathrm{H} 20 \mathrm{D}$, indicating that, despite not showing significant differences in the 229 number of BrdU+ cells per crypt (figure 1J), H10D already generates a change in the proliferation 230 behavior of the ISCs.

\section{Acute HBOT does not change enzymatic activity in the small intestine}

232 Intestines from H20D did not show differences in comparison to their control in the enzymatic activity 233 related to oxidative metabolism. We sought to analyze if the increment in the proliferation of the ISCs 234 by HBOT could be explained by an increase in the activity of cytochrome c oxidase (COX) and citrate 235 synthase (CS), both enzymes related with aerobic ATP generation. To this end, we analyzed the 236 activity of these enzymes in the whole duodenum in each experimental group (figure $3 \mathrm{~A}$ ). No 237 difference was found in the activity of both enzymes, when analyzed per gram, total in tissue or total 238 protein between control (C10D and C20D) and both HBOT groups (figure 3B-C). Therefore, HBOT 239 effects must encompass another molecular pathway to account for its effect.

240 HBOT modulates proliferation of the ISCs through mTORC1 pathway activation

241 To evaluate if HBOT could activate the mTOR pathway, capable of sensing changes in oxygen levels, 242 we generated four groups, combining rapamycin with or without HBOT (see table II). In line with 243 the literature, animals treated with rapamycin showed a mTORc1 pathway suppression as revealed 244 by the lack of phosphorylation on S6K1 (figure 4A; supplementary figure 1). On the other hand, 245 animals injected with rapamycin vehicle (DMSO) showed no inhibition on the phosphorylation status 246 of S6K1, either with or without HBOT. Stunningly, animals that received a combined treatment with 247 rapamycin and HBOT revealed strong phosphorylation on S6K1 (Figure 4B). Therefore, we suggest 248 that HBOT can recover rapamycin inhibition of mTOR pathway signaling. 
249 Acute HBOT can simulate the effects on ISCs proliferation generated by a CR. When we compared 250 the effects on ISCs proliferation of rapamycin (Rapa Ctrl) and HBOT for 20 days (DMSO HBOT), 251 we found that each treatment increases the number of BrdU+ cells per crypt (figure 5 A-E). 252 Nevertheless, animals treated with both HBOT and rapamycin (Rapa HBOT) show no increase in 253 ISC proliferation.

254 Finally, a careful analysis of frequency distribution of BrdU+ cells per crypt indicated that only 2 255 comparisons present no differences. Rapa HBOT and DMSO Ctrl groups exhibited no differences 256 (figure 6F) while Rapa Ctrl and DMSO HBOT groups also showed no differences in their 257 distributions (figure 6G). These data, in combination with the results presented in figure 5E, suggest 258 that HBOT has the same effects on ISCs proliferation as a CR generated by rapamycin. 


\section{Discussion}

272 HBOT has been reported to modulate the stem cells proliferative behavior in two well-known adult

273 stem cell niches, in the bone marrow (Geng et al; 2015) and the brain (Wang et al; 2007). In this

274 work, we demonstrate that HBOT also can enhance the proliferation of ISCs in a synchronous fashion.

275 Moreover, we provide findings that this proliferative effect is mediated by mTORc1 signaling.

276 Nearly $90 \%$ of the intestinal epithelium is replaced every 3-4 days by cells newly generated from the

277 crypt epithelium. However, long-lived ISCs harbor in the crypt bottom to replenish the large amount

278 of disposable functional epithelium. ISCs produce rapidly cycling progenitor cells that migrate up the

279 crypt-villus axis and differentiate into mature epithelial cells that are eventually shed off into the

280 lumen. Small intestine partial pressure of oxygen (PO2) values have been reported to be 2-5\%, 3-

$2816 \%$ and 5-9\% O2 for lumen, mucosa and serosa layers, respectively (Espey; 2013).

282 To explore whether oxygen tension impacts cellular turnover in the small intestine, we used an 283 established HBOT protocol (Peña-Villalobos et al; 2018). In our previous work, rodents were 284 exposed to HBOT conditions in ten consecutive sessions. We did not find a significant effect of 285 HBOT on cell proliferation, as all groups had similar numbers of BrdU+ cells per crypt. But in the 286 present work, increasing the time length of treatment by applying HBOT for 20 consecutive days lead 287 to a significant cell proliferation rise within the crypts. The effect of HBOT is clearly observable on day 20 but had an accumulative effect that was already evidenced since day 10 . The analysis of the frequency distribution of BrdU+ positive cells from C10D with H10D groups indicates that despite showing no differences in the number of BrdU+ cells per crypts (figure 1J), there are differences in

291 the frequency of proliferative cells (figure 2). The latter indicating that HBOT for 10 consecutive 292 days already generates a change in the proliferation dynamics of the ISCs within the crypts.

293 Using the specific ISCs marker, Olfm4, we observed that stem cells co-distribute with the 294 proliferation marker BrdU. Of note, another cell population shows proliferative labeling but no 295 Olfm4 staining (figure 1E). Thus, we cannot discard the possibility that HBOT can stimulate 
296 proliferation of progenitor cells and/or emergency ISCs. The recruitment of these cells together with 297 ISCs would represent an interesting broader effect of HBOT. Keeping in mind that HBOT has 298 recently reported to increase the proliferation of progenitor cells in vitro (Benincasa et al; 2019), our 299 result would be the first in describing similar results in vivo in the intestinal niche. On the other hand, 300 if, in addition, the target of acute HBOT are the so-called emergency ISCs, a population that has been 301 described as quiescent (Muñoz et al; 2012), this could mean that the treatment can promote their 302 recruitment to ISCs. The possible relationship between the quiescent and the actively cycling ISCs 303 and the biological consequences of their HBOT response needs to be further explored.

304 The increase in proliferation in H20D occurred in a synchronous fashion (figure 1K). This means that 305 along the duodenum all the H20D crypts presented a similar and higher number of BrdU+ cells than 306 the control or H10D groups. This synchronicity could emerge as a consequence of cell cycle arrest 307 provoked by HBOT or by the maximization of the proliferative capacity of the crypt's cells. As shown 308 by previous authors, identification of the BrdU and PHH3 proportion allows for evaluation of cell 309 cycle arrest (Cappella et al; 2008 and Ren et al; 2018). Based on these works we analyzed the 310 proportion of the aforementioned markers in the C20D and H20D groups and determined that HBOT 311 does not arrest the cells in a particular stage of the cell cycle. Thus, HBOT maximizes the proliferation 312 mode in the intestinal crypts.

313 It is critical to note that these effects were not generated by an increase in ATP production due to an 314 enhanced oxidative metabolism by an increment in available oxygen, as revealed by the analysis of 315 the enzymatic activity of COX and CS (figure 3 B-C). These results were in concordance with our 316 previous work, where we showed that using HBOT for 10 consecutive days did not change the basal 317 metabolic rates of mice (Peña-Villalobos et al; 2018). They are also in line with a recent in vitro 318 analysis of lung fibroblast where no changes in CS activity were reported (Dejmek et al; 2018). 319 Notwithstanding, it is remarkable how a treatment that increases oxygen tension does not alter 320 oxidative metabolism in the intestinal epithelium while other reports indicate that acute HBOT does 
321 generate changes in tissues like brain or skeletal muscle (Matchett et al; 2009 and Ishihara; 2019). If

322 HBOT can generate a differential physiological response in tissues and organs warrants further

323 investigation.

324 mTORC1 is known to sense oxygen levels (Lane et al; 2017) and to regulate stem cell proliferation

325 (Murakami et al; 2004 and Hu et al; 2017). The stimulation of S6K1 by mTORC1 has been widely

326 reported. In addition, mTORC1 has been characterized as a rapamycin sensitive complex. Strikingly,

327 we found that HBOT can recover rapamycin induced mTOR pathway inhibition, as shown in figure

$3284 \mathrm{~A}$ and supplementary figure 1. Furthermore, analysis of BrdU+ cells per crypt showed that HBOT

329 and rapamycin increased proliferation of the cells within the crypt independently. We attribute this

330 effect of rapamycin to its capacity to mimic a CR. Under CR, Paneth cells, forming part of the stem

331 cell niche, sense a downregulation of mTORC1 and generate a paracrine signal (NAD synthesis)

332 stimulating ISCs to proliferate (Igarashi \& Guarente; 2016 and Peña-Villalobos et al; 2019). HBOT

333 also increases proliferation through mTORC1, but, at this point, we would like to suggest that it

334 probably occurs through a different and competitive way of rapamycin's action. When both

335 treatments are combined, there is no increase or synergic effect between them. On the contrary, they

336 cancel each other's effects (figure 4), resulting in no differences in the number of BrdU+ cells per

337 crypt and the frequency distribution of ISCs (figure 5A, 6F). Therefore, HBOT can modulate the

338 proliferation of ISCs (and most likely progenitor cells) in the crypt acting through mTOR pathway

339 signaling. The possible intricate network of the interplay between these two metabolic mTORC1

340 stimuli with similar output warrants further investigation.

341 The intrinsic metabolism of adult stem cells that reside in a hypoxic condition like neural stem cells

342 in the subventricular zone or mesenchymal stromal cells in the bone marrow seems to be maintained

343 by an aerobic glycolysis but without generating high levels of reactive oxygen species (ROS) and

344 therefore maintaining a low proliferation rate that preserves the self-renewal potential (Shyh-Chang

$345 \& \mathrm{Ng} ; 2017)$. But when these cells sense a high ROS environment, they undergo an active 
347 hyperproliferation in response to high ROS levels (Hochmuth et al; 2011). This high ROS level can 348 be produced by an increase in mitochondrial activity which in turn can be activated by an increase in 349 mTOR signaling. On the other hand, it has been proposed that changes in the REDOX state of stem 350 cells, induced by changes in the electron transport chain due oxygen reduction, can change their 351 proliferative behavior and might have effects at nuclear epigenetic and gene expression (Wei et al; 352 2018). Therefore, the increase in ISCs proliferation in mice could be explained by a response to an 353 increase in ROS levels or changes in REDOX state. Our group is currently investigating the 354 relationship between HIF1- $\alpha$, ROS levels and mTORC1 as a candidate mechanism to explain the 355 effects in response to hyperbaric oxygen.

356 The metabolic program of stem cells is intrinsically determined and essential for the maintenance of 357 those cells, impacting the balance of self- renewal, and differentiation. HBOT has positive effects in 358 bone marrow stem cells migration and mesenchymal stem cells proliferation (Thom et al; 2006 and 359 Geng et al; 2015). Here we show that HBOT can be also used to increase the proliferation of ISCs 360 and progenitor cells through mTORc1 pathway signaling, without altering the oxidative metabolism 361 of the animal's small intestine. Results in line with recent findings pointing to the capacity of 362 mTORc1 to regulate the proliferation state of post-natal skeletal stem cells (Newton et al; 2019). Most 363 likely, since HBOT promotes a systemic effect and other tissue-resident stem cell pools are affected. 364 Future studies need to address a possible HBOT/mTORC1 signaling on other somatic stem cells 365 populations.

366 In summary, our findings indicate that HBOT on its own can mimic the effects of rapamycin on 367 proliferation of ISCs. Noteworthy, HBOT has the advantage of being a non-invasive and relatively 368 cheap treatment, favoring its use in future therapies to regenerate damaged intestinal epithelia. 


\section{Conclusions}

371 HBOT for 20 consecutive days potentiates ISC proliferative behavior, such increase in proliferation

372 occurs in a synchronous fashion all along the duodenum without altering oxidative metabolism, 373 suggesting that HBOT maximizes the proliferation rate of ISC. Our results show that mTORC1 374 pathway modulation is responsible for this HBOT driven increase in proliferation. When mTORC1 375 is inhibited by rapamycin, ISCs undergo a caloric restriction like situation, that in mice generates 376 similar effect to that observed if they had been treated with HBOT for 20 consecutive days. However, 377 when both treatments were combined, no effect on ISC proliferation were detected, suggesting that 378 HBOT effect were modulates through mTORC1 pathway, and due its inhibition, the effect disappears. 379 These results shed light on the molecular mechanisms underlying HBOT therapeutic action, a medical 380 treatment that has been used for a long time but whose subjacent mechanisms remain unclear.

\section{Acknowledgments}

382 The authors would like to acknowledge Osorio Hermanos \& Cia for fabrication and maintenance of the experimental HBOT chamber, also to Dr. Manuel Varas for providing the S6K1antibody. 


\section{References}

386 Barker, N. (2014). Adult intestinal stem cells: critical drivers of epithelial homeostasis and regeneration. Nature reviews Molecular cell biology, 15(1), 19-33.

Basak, O., Beumer, J., Wiebrands, K., Seno, H., van Oudenaarden, A., \& Clevers, H. (2017). Induced quiescence of Lgr5+ stem cells in intestinal organoids enables differentiation of hormone-producing enteroendocrine cells. Cell stem cell, 20(2), 177-190.

Benincasa, J. C., de Freitas Filho, L. H., Carneiro, G. D., Sielski, M. S., Giorgio, S., Werneck, C. C., \& Vicente, C. P. (2019). Hyperbaric oxygen affects endothelial progenitor cells proliferation in vitro. Cell biology international, 43(2), 136-146.

Benjamin, D., Colombi, M., Moroni, C., \& Hall, M. N. (2011). Rapamycin passes the torch: a new generation of mTOR inhibitors. Nature reviews Drug discovery, 10(11), 868.

Bennett, M. H., Trytko, B., \& Jonker, B. (2012). Hyperbaric oxygen therapy for the adjunctive treatment of traumatic brain injury. Cochrane Database of Systematic Reviews, (12).

Cappella, P., Gasparri, F., Pulici, M., \& Moll, J. (2008). A novel method based on click chemistry, which overcomes limitations of cell cycle analysis by classical determination of BrdU incorporation, allowing multiplex antibody staining. Cytometry Part A: the journal of the International Society for Analytical Cytology, 73(7), 626-636.

Clevers, H. (2013). The intestinal crypt, a prototype stem cell compartment. Cell, 154(2), 274-284.

Cox, L. S., \& Mattison, J. A. (2009). Increasing longevity through caloric restriction or rapamycin feeding in mammals: common mechanisms for common outcomes? Aging Cell, 8(5), 607-613.

Dai, D. F., Karunadharma, P. P., Chiao, Y. A., Basisty, N., Crispin, D., Hsieh, E. J., ... \& Beyer, R. P. (2014). Altered proteome turnover and remodeling by short-term caloric restriction or rapamycin rejuvenate the aging heart. Aging cell, 13(3), 529-539.

Dave, K. R., Prado, R., Busto, R., Raval, A. P., Bradley, W. G., Torbati, D., \& Perez-Pinzon, M. A. (2003). Hyperbaric oxygen therapy protects against mitochondrial dysfunction and delays onset of motor neuron disease in Wobbler mice. Neuroscience, 120(1), 113-120.

De Mey, J. R., \& Freund, J. N. (2013). Understanding epithelial homeostasis in the intestine: an old battlefield of ideas, recent breakthroughs and remaining controversies. Tissue barriers, 1(2), e24965.

Dejmek, J., Kohoutova, M., Kripnerova, M., Čedíková, M., Tůma, Z., Babuška, V., ... \& Kuncova, J. (2018). Repeated exposure to hyperbaric hyperoxia affects mitochondrial functions of the lung fibroblasts. Physiological Research, 67.

Dhar, M., Neilsen, N., Beatty, K., Eaker, S., Adair, H., \& Geiser, D. (2012). Equine peripheral bloodderived mesenchymal stem cells: isolation, identification, trilineage differentiation and effect of hyperbaric oxygen treatment. Equine veterinary journal, 44(5), 600-605.

Eskes, A. M., Ubbink, D. T., Lubbers, M. J., Lucas, C., \& Vermeulen, H. (2011). Hyperbaric oxygen therapy: solution for difficult to heal acute wounds? Systematic review. World journal of surgery, 35(3), 535-542. 
Espey, M. G. (2013). Role of oxygen gradients in shaping redox relationships between the human intestine and its microbiota. Free Radical Biology and Medicine, 55, 130-140.

Fingar, D. C., Richardson, C. J., Tee, A. R., Cheatham, L., Tsou, C., \& Blenis, J. (2004). mTOR controls cell cycle progression through its cell growth effectors S6K1 and 4E-BP1/eukaryotic translation initiation factor 4E. Molecular and cellular biology, 24(1), 200-216.

Fingar, D. C., Salama, S., Tsou, C., Harlow, E. D., \& Blenis, J. (2002). Mammalian cell size is controlled by mTOR and its downstream targets S6K1 and 4EBP1/eIF4E. Genes \& development, 16(12), 1472-1487.

Geng, C. K., Cao, H. H., Ying, X., \& Yu, H. L. (2015). Effect of mesenchymal stem cells transplantation combining with hyperbaric oxygen therapy on rehabilitation of rat spinal cord injury. Asian Pacific journal of tropical medicine, 8(6), 468-473.

Hochmuth, C. E., Biteau, B., Bohmann, D., \& Jasper, H. (2011). Redox regulation by Keap1 and Nrf2 controls intestinal stem cell proliferation in Drosophila. Cell stem cell, 8(2), 188-199.

Hu, J. K. H., Du, W., Shelton, S. J., Oldham, M. C., DiPersio, C. M., \& Klein, O. D. (2017). An FAKYAP-mTOR signaling axis regulates stem cell-based tissue renewal in mice. Cell stem cell, 21(1), 91-106.

Igarashi, M., \& Guarente, L. (2016). mTORC1 and SIRT1 cooperate to foster expansion of gut adult stem cells during calorie restriction. Cell, 166(2), 436-450.

Ishihara, A. (2019). Mild hyperbaric oxygen: mechanisms and effects. The Journal of Physiological Sciences, 69(4), 573-580.

Kalani, M., Jörneskog, G., Naderi, N., Lind, F., \& Brismar, K. (2002). Hyperbaric oxygen (HBO) therapy in treatment of diabetic foot ulcers: long-term follow-up. Journal of Diabetes and its Complications, 16(2), 153-158.

Kranke, P., Bennett, M. H., Martyn-St James, M., Schnabel, A., Debus, S. E., \& Weibel, S. (2015). Hyperbaric oxygen therapy for chronic wounds. Cochrane Database of Systematic Reviews, (6).

Lane, J. D., Korolchuk, V. I., Murray, J. T., Rabanal-Ruiz, Y., \& Otten, E. G. (2017). mTORC1 as the main gateway to autophagy. Essays in biochemistry, 61(6), 565-584.

Lee, C. C., Chen, S. C., Tsai, S. C., Wang, B. W., Liu, Y. C., Lee, H. M., \& Shyu, K. G. (2006). Hyperbaric oxygen induces VEGF expression through ERK, JNK and c-Jun/AP-1 activation in human umbilical vein endothelial cells. Journal of biomedical science, 13(1), 143-156.

Lehner, B., Sandner, B., Marschallinger, J., Lehner, C., Furtner, T., Couillard-Despres, S., ... \& Aigner, L. (2011). The dark side of BrdU in neural stem cell biology: detrimental effects on cell cycle, differentiation and survival. Cell and tissue research, 345(3), 313.

Matchett, G. A., Martin, R. D., \& Zhang, J. H. (2009). Hyperbaric oxygen therapy and cerebral ischemia: neuroprotective mechanisms. Neurological Research, 31(2), 114-121.

Muñoz, J., Stange, D. E., Schepers, A. G., Van De Wetering, M., Koo, B. K., Itzkovitz, S., ... \& Myant, K. (2012). The Lgr5 intestinal stem cell signature: robust expression of proposed quiescent '+ 4'cell markers. The EMBO journal, 31(14), 3079-3091. 
Murakami, M., Ichisaka, T., Maeda, M., Oshiro, N., Hara, K., Edenhofer, F., ... \& Yamanaka, S. (2004). mTOR is essential for growth and proliferation in early mouse embryos and embryonic stem cells. Molecular and cellular biology, 24(15), 6710-6718.

Newton, P. T., Li, L., Zhou, B., Schweingruber, C., Hovorakova, M., Xie, M., ... \& Suter, S. (2019). A radical switch in clonality reveals a stem cell niche in the epiphyseal growth plate. Nature, 567(7747), 234-238.

Peña-Villalobos, I., Casanova-Maldonado, I., Lois, P., Prieto, C., Pizarro, C., Lattus, J., ... \& Palma, V. (2018). Hyperbaric oxygen increases stem cell proliferation, angiogenesis and wound-healing ability of WJ-MSCs in diabetic mice. Frontiers in physiology, 9, 995.

Peña-Villalobos, I., Casanova-Maldonado, I., Lois, P., Sabat, P., \& Palma, V. (2019). Adaptive physiological and morphological adjustments mediated by intestinal stem cells in response to food availability in mice. Frontiers in physiology, 9, 1821.

Ren, J., Tang, C. Z., Li, X. D., Niu, Z. B., Zhang, B. Y., Zhang, T., ... \& Wang, F. C. (2018). Identification of $\mathrm{G} 2 / \mathrm{M}$ phase transition by sequential nuclear and cytoplasmic changes and molecular markers in mice intestinal epithelial cells. Cell Cycle, 17(6), 780-791.

Sampson, L. L., Davis, A. K., Grogg, M. W., \& Zheng, Y. (2015). mTOR disruption causes intestinal epithelial cell defects and intestinal atrophy postinjury in mice. The FASEB Journal, 30(3), 12631275.

Saxton, R. A., \& Sabatini, D. M. (2017). mTOR signaling in growth, metabolism, and disease. Cell, 168(6), 960-976.

Schieke, S. M., Phillips, D., McCoy, J. P., Aponte, A. M., Shen, R. F., Balaban, R. S., \& Finkel, T. (2006). The mammalian target of rapamycin (mTOR) pathway regulates mitochondrial oxygen consumption and oxidative capacity. Journal of Biological Chemistry, 281(37), 27643-27652.

Shyh-Chang, N., \& Ng, H. H. (2017). The metabolic programming of stem cells. Genes \& development, 31(4), 336-346.

Snippert, H. J., Van Der Flier, L. G., Sato, T., Van Es, J. H., Van Den Born, M., Kroon-Veenboer, C., ... \& Clevers, H. (2010). Intestinal crypt homeostasis results from neutral competition between symmetrically dividing Lgr5 stem cells. Cell, 143(1), 134-144.

Sunkari, V. G., Lind, F., Botusan, I. R., Kashif, A., Liu, Z. J., Ylä-Herttuala, S., ... \& Catrina, S. B. (2015). Hyperbaric oxygen therapy activates hypoxia-inducible factor 1 (HIF-1), which contributes to improved wound healing in diabetic mice. Wound Repair and Regeneration, 23(1), 98-103.

Thom, S. R., Bhopale, V. M., Velazquez, O. C., Goldstein, L. J., Thom, L. H., \& Buerk, D. G. (2006). Stem cell mobilization by hyperbaric oxygen. American Journal of Physiology-Heart and Circulatory Physiology.

Totafurno, J. O. H. N., Bjerknes, M., \& Cheng, H. A. Z. E. L. (1987). The crypt cycle. Crypt and villus production in the adult intestinal epithelium. Biophysical journal, 52(2), 279-294.

Tothova, Z., Kollipara, R., Huntly, B. J., Lee, B. H., Castrillon, D. H., Cullen, D. E., ... \& Armstrong, S. A. (2007). FoxOs are critical mediators of hematopoietic stem cell resistance to physiologic oxidative stress. Cell, 128(2), 325-339. 
Wang, T., Tsui, B., Kreisberg, J. F., Robertson, N. A., Gross, A. M., Yu, M. K., ... \& Ideker, T. (2017). Epigenetic aging signatures in mice livers are slowed by dwarfism, calorie restriction and rapamycin treatment. Genome biology, 18(1), 57.

Wang, X. L., Yang, Y. J., Xie, M., Yu, X. H., Liu, C. T., \& Wang, X. (2007). Proliferation of neural stem cells correlates with Wnt-3 protein in hypoxic-ischemic neonate rats after hyperbaric oxygen therapy. Neuroreport, 18(16), 1753-1756.

Wei, P., Dove, K. K., Bensard, C., Schell, J. C., \& Rutter, J. (2018). The force is strong with this one: metabolism (over) powers stem cell fate. Trends in cell biology, 28(7), 551-559.

Wei, Y., Yu, L., Bowen, J., Gorovsky, M. A., \& Allis, C. D. (1999). Phosphorylation of histone H3 is required for proper chromosome condensation and segregation. Cell, 97(1), 99-109.

Yilmaz, Ö. H., Katajisto, P., Lamming, D. W., Gültekin, Y., Bauer-Rowe, K. E., Sengupta, S., ... \& Nielsen, G. P. (2012). mTORC1 in the Paneth cell niche couples intestinal stem-cell function to calorie intake. Nature, 486(7404), 490.

Zhang, T., Yang, Q. W., Wang, S. N., Wang, J. Z., Wang, Q., Wang, Y., \& Luo, Y. J. (2010). Hyperbaric oxygen therapy improves neurogenesis and brain blood supply in piriform cortex in rats with vascular dementia. Brain injury, 24(11), 1350-1357.

Zhang, X. Y., Yang, Y. J., Xu, P. R., Zheng, X. R., Wang, Q. H., Chen, C. F., \& Yao, Y. (2011). The role of $\beta$-catenin signaling pathway on proliferation of rats neural stem cells after hyperbaric oxygen therapy in vitro. Cellular and molecular neurobiology, 31(1), 101-109.

(1)

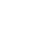

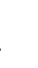

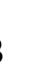

4

5

26

27

28

9

30

31


533 Declarations

534

535 Consent for publication: Not applicable

536 Availability of data and material: All data is available as figures or supplementary information

537 Funding: Work was supported by FONDEF grant D09E1047 (VP), FONDECYT Postdoctoral

5383180108 (IPV) and FONDECYT grant 1110237 and 1140697 (VP).

$539 \quad$ Author contributions

540 Conceptualization: ICM, VP \& IPV

541 Investigation: ICM, IPV \& PL

542 Formal Analysis: ICM \& IPV

543 Writing-Original Draft: ICM; IPV \& DA

544 Writing-Review \& Editing: VP \& PL

545 Supervision: VP

546 Declaration of interests: The authors declare no competing financial interests.

547 Ethical standards: All procedures performed to elaborate this manuscript comply with the Chilean

548 legislation and were approved by Institutional and Bioethical Use Committees (University of Chile). 


\section{Figures}
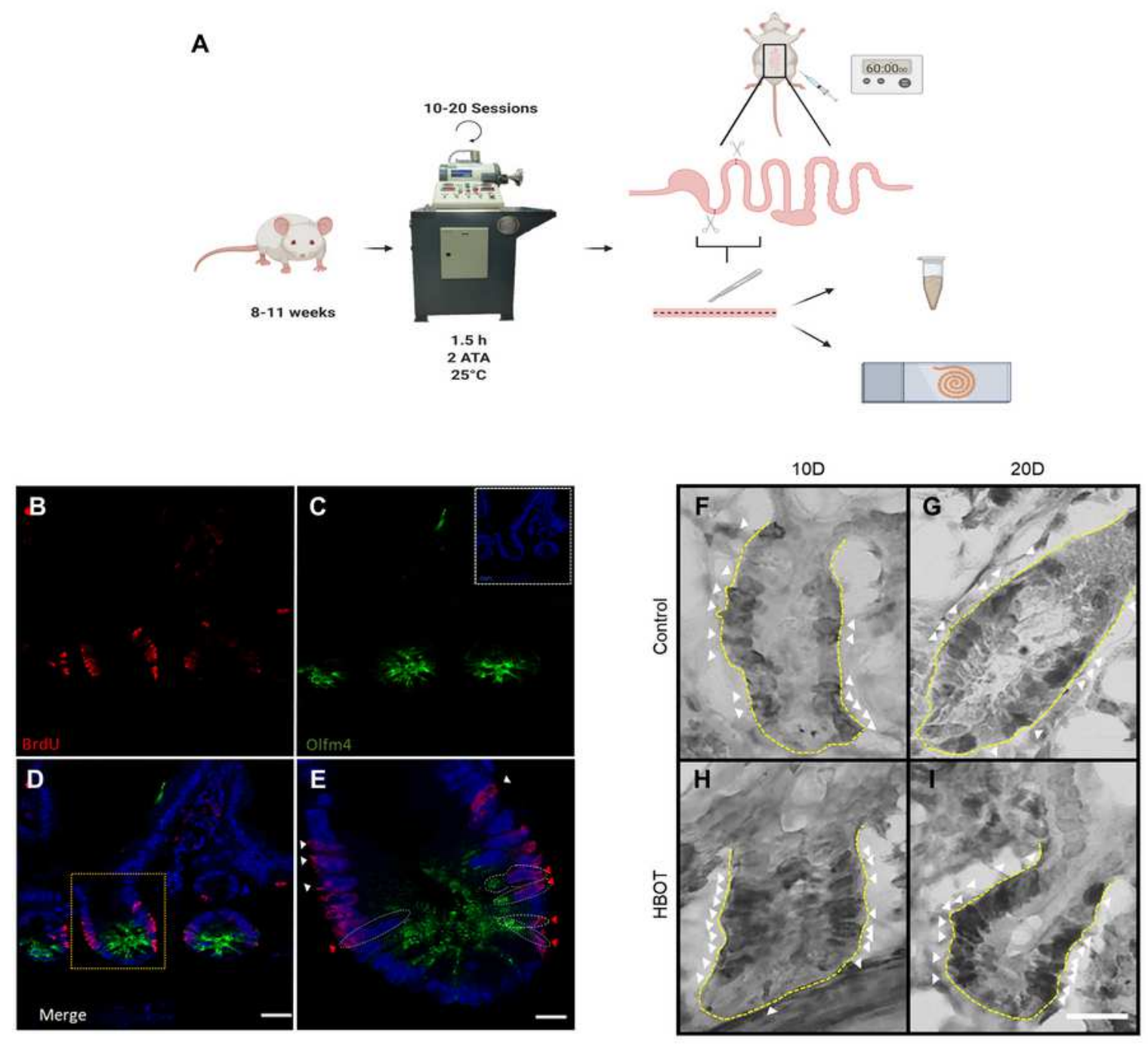

$\mathbf{J}$

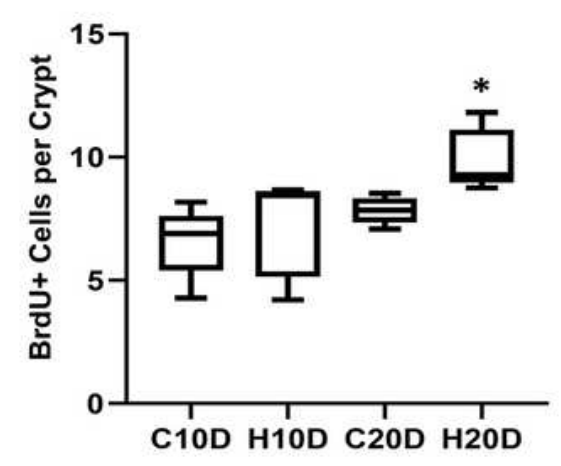

K

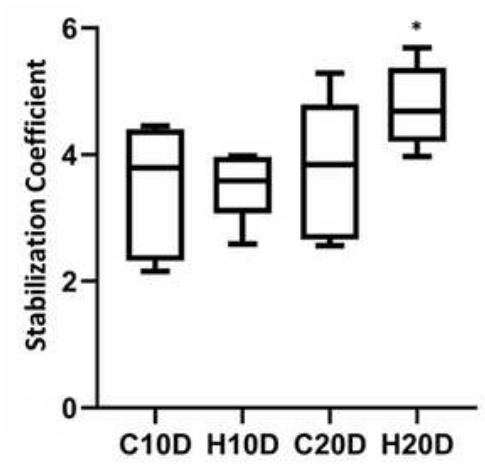

Figure 1

HBOT increases ISCs proliferation in a synchronous fashion. A) Schematic workflow of HBOT treatment and experimental procedures on small intestine samples. B-E) Representative immunofluorescence images of ISC proliferation in intestinal crypts. B) Proliferative cells were detected by BrdU labeling (Red). 
C) ISCs labeled as Olfm4+ cells, a cytoplasmatic marker (Green). Inset shows DAPI nuclear staining (Blue). D) Merged image. Yellow box indicates crypt with proliferating ISCs among other cell populations. Bar= $15 \mathrm{gm}$. E) Zoom of yellow box from image D. Red arrowheads indicate proliferating ISCs while white arrowheads indicate other proliferating cells within the intestinal crypt. Bar= $30 \mathrm{gm}$. F-G) Representative immunohistochemistry images of intestinal crypts with proliferating BrdU+ cells; experimental groups as indicated. Nuclei labeled by hematoxylin counterstaining. Bar= $20 \mathrm{gm}$. Yellow dotted lines outline the crypts while white arrowheads show BrdU+ cells. 1) Quantification of BrdU+ cells per crypt for each treatment. Asterisk indicate statistical significance. Permutation test. pc 0.05. n=5. K) Stabilization coefficient indicating the level of variation inside each group. Asterisk indicates statistical significance. Only H2OD shows significant differences, indicating synchronicity. Permutation test. $p<0.05 . n=4$.

\section{C $10 \mathrm{D}$}

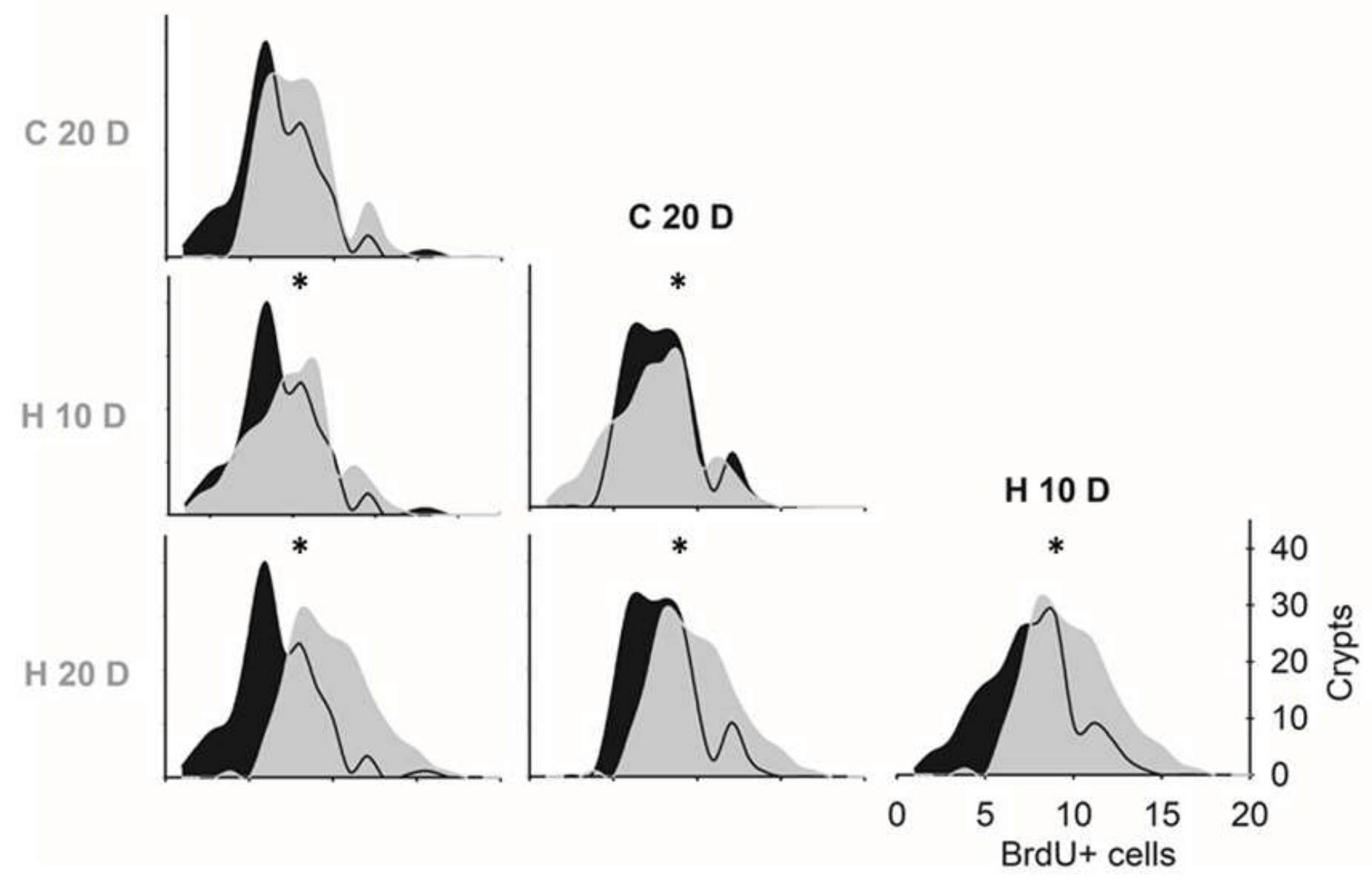

Figure 2

HBOT has an accumulative effect on time. Comparison of frequency distribution of Bra], cells per crypt between groups as indicated. Asterisk indicates differences $(p<0,05)$. Kohnogorov-Smimov test. Only the comparison between control groups shows no differences. $n=4$. 

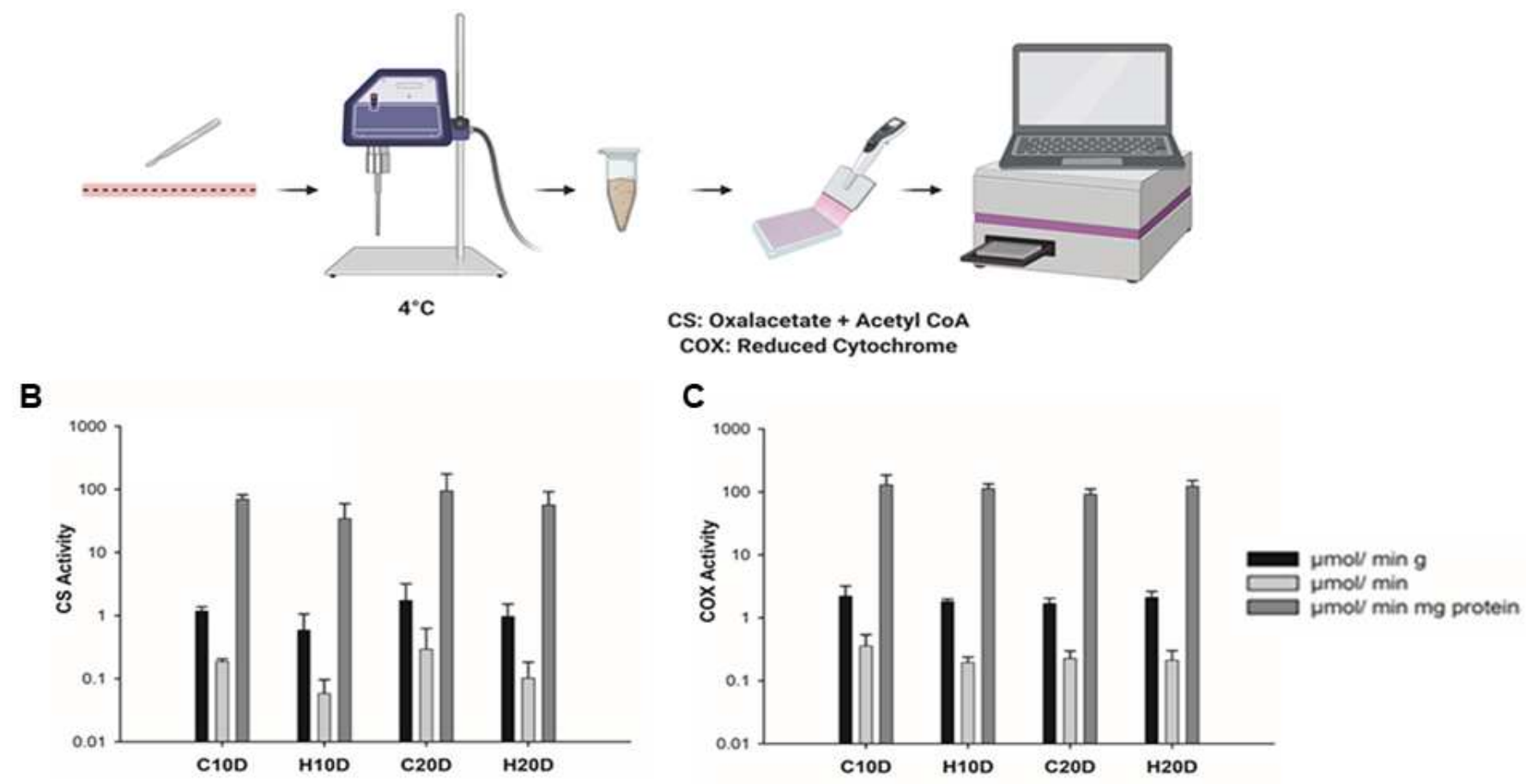

\section{Figure 3}

Aga. SNOT does not affect enzyme. activity In the small Intestine. A) Woncilow of enzymatic assay Cr CS sod COX activity in small inhffine tissue. 13) Bar graph of CS activity finnoknin) under each trealment. No significant differences were found. ( $p>0.05$ ). nw 3. C) COX activity (pmoUmin) under each treatment. No significant differences were found. Black bars indicate activity per gram of tissue, dark grey bars indicate activity per total proteins in the sample and tight grey bars indicate total activity. (pe 0.135$) . n=3$.

A

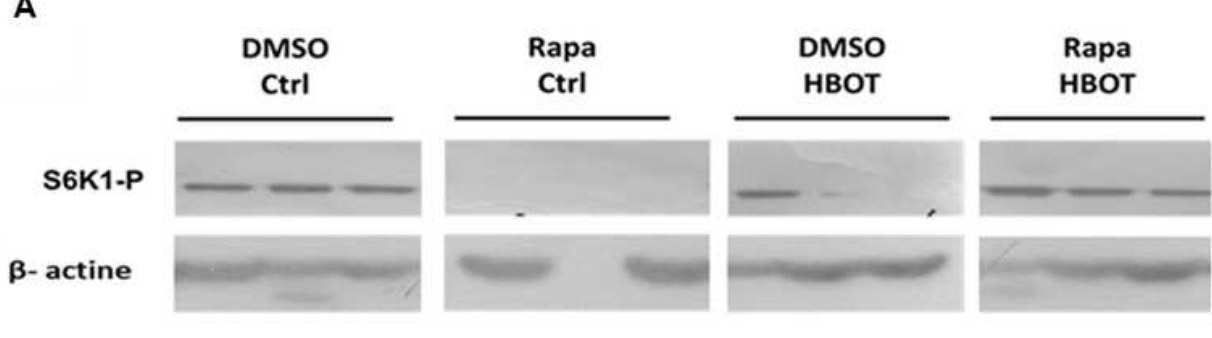

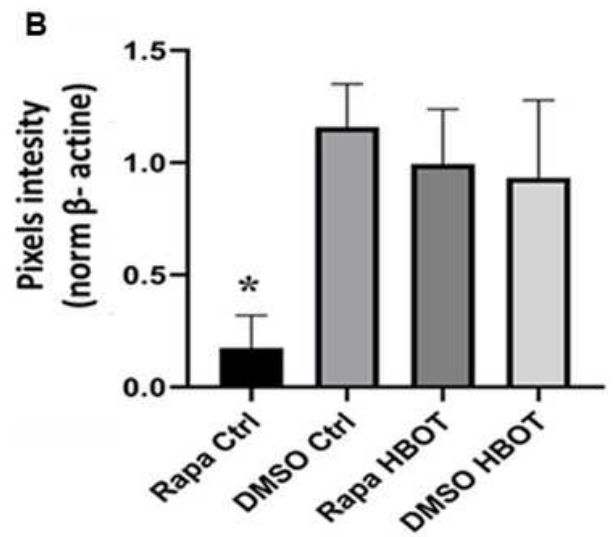

Figure 4

HB07 renovers rapamycin induced mTOR pathway inhibition. A) Western blot for phosphorylated S6101 (SliK1.14) and 13-actine in each experimental group (triplicate). First row shows DMSO control group. Second row shows rapamycin control group. Third row shows rapamycin and HOOT group. Last row 
shows DMSO and MOT group. SPOT sessions for 20 days. B) Quantification of pixel Wensity for SCr141$P$ presence in each group normalized with internal control p.n.s. Asterisks indicates statistical significance. Permutation hst.p<0.05, r, 2 per grow.
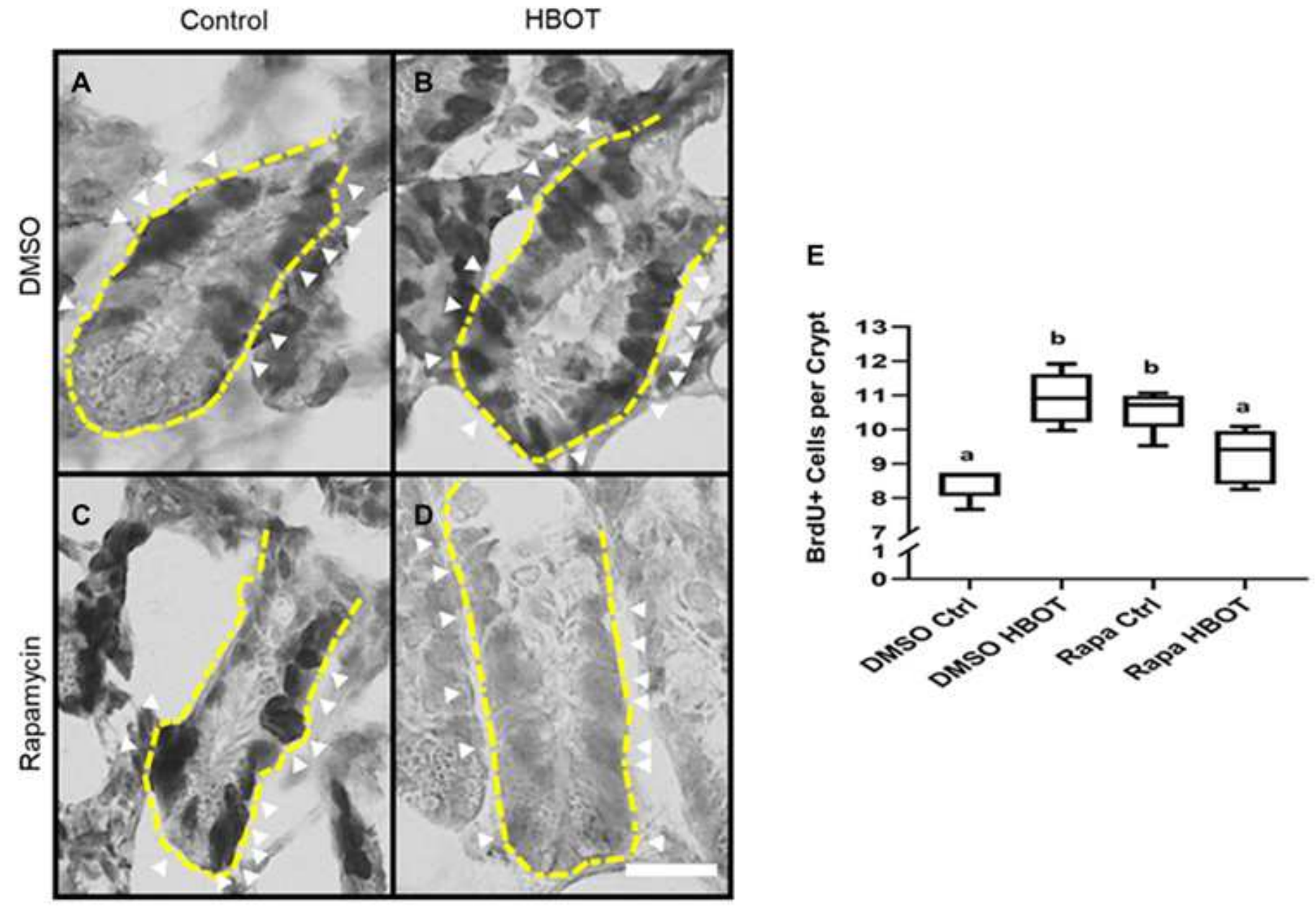

Figure 5

HBOT and rapamyelo increase ISCs proliferation independently. A-D) Representative immunobistochemistly images for proliferating cells. label. by Brdu incorporation. in intestinal crypts for each experimental group. 20 days of treatment in each group. Nuclei labeled by heinatoxylin cramterstaining. Bar- 20 inn. Yellow dotted lines outline the crypt periphay. White arrowhinids show Brdfft E) Quantification of Brdlit cells for each treatment Same letters indicate no statinical significance while different letters indicate statistical significance. PermuNtion test. $p<0.05$. 11-5 per group. 

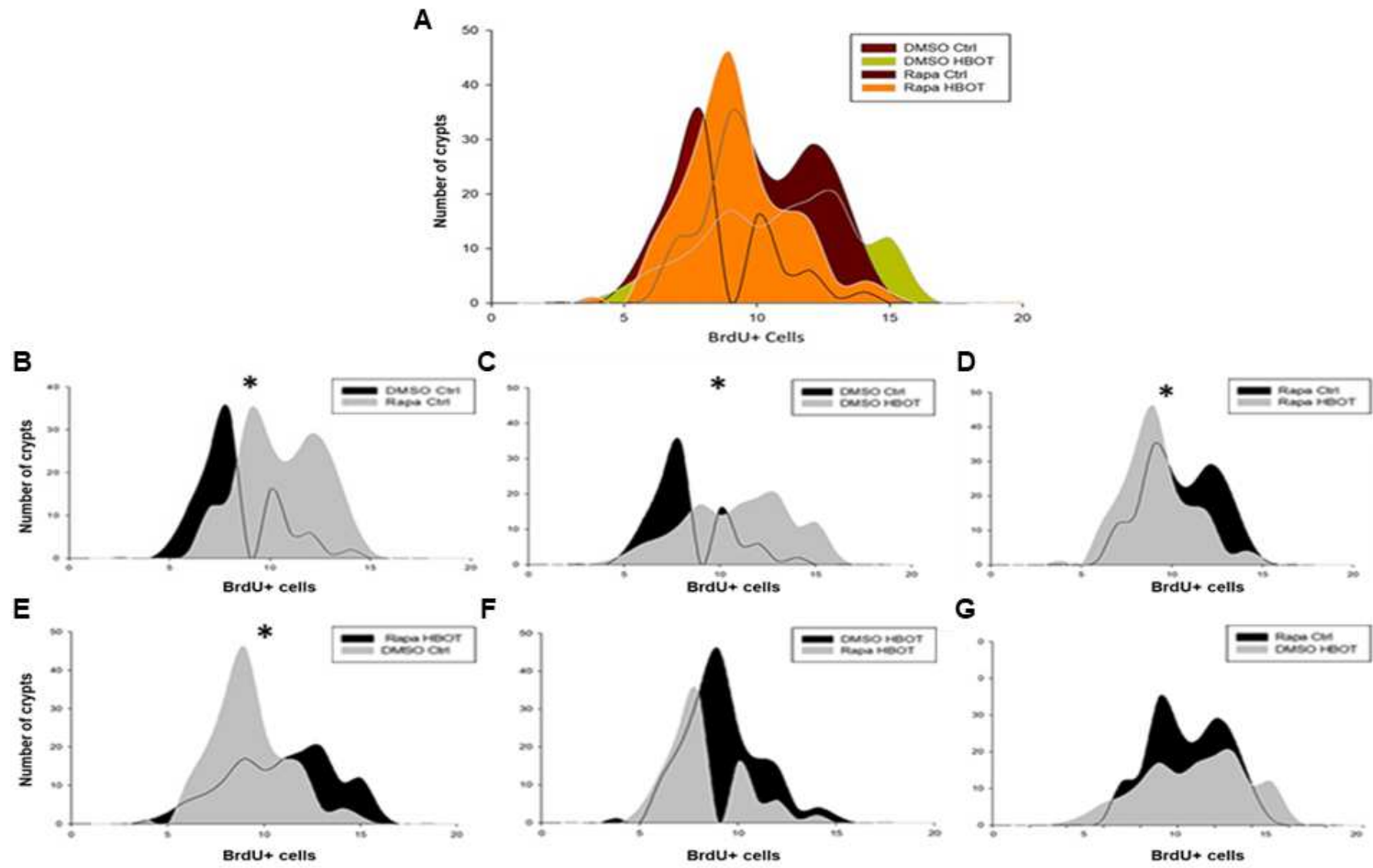

Figure 6

HBDE can simulate a caloric restriction. A) Superposition of frequency distribution for each Reap. All comparison was made by a KolmogorovsSmirnov test. B) Comparison between DMSO CPI and Rape Cid. pm 0.004 C) Comparison between DMSO CU) and DMSO 1180T. m 0.003 D) Comparison between Rape CPI and Raper BOOT. m 0.013 E) Comparison between DMSO Cirl and Rape BOOT. 0.009 r) Comparison between Rape 11BOT and DMSO Ctd. p>0.05 G) Comparison between Raper Crd end DMSO 11BOT. $p>0.05 . n=5$.

\section{Supplementary Files}

This is a list of supplementary files associated with this preprint. Click to download.

- SF1.tif 